\title{
Matrix-free proof of a regularity characterization
}

\author{
A. Czygrinow \\ Department of Mathematics and Statistics \\ Arizona State University, Tempe, Arizona 85287, USA \\ andrzej@math.la.asu.edu \\ B. Nagle \\ Department of Mathematics and Statistics \\ University of Nevada, Reno, Nevada 89557, USA \\ nagle@unr.edu
}

Submitted: May 28, 2003; Accepted: Oct 7, 2003; Published: Oct 13, 2003

MR Subject Classifications: 05C35, 05C80

\begin{abstract}
The central concept in Szemerédi's powerful regularity lemma is the so-called $\varepsilon$-regular pair. A useful statement of Alon et al. essentially equates the notion of an $\varepsilon$-regular pair with degree uniformity of vertices and pairs of vertices. The known proof of this characterization uses a clever matrix argument.

This paper gives a simple proof of the characterization without appealing to the matrix argument of Alon et al. We show the $\varepsilon$-regular characterization follows from an application of Szemerédi's regularity lemma itself.
\end{abstract}

\section{Introduction}

The well-known Szemerédi Regularity Lemma [7] (cf. [4] or [5]) may be the single most powerful tool in extremal graph theory. Roughly speaking, this lemma asserts that every large enough graph may be decomposed into constantly many "random-like" induced bipartite subgraphs (i.e. " $\varepsilon$-regular pairs"). A property of the $\varepsilon$-regular pairs obtained from Szemerédi's lemma is studied in this note.

Suppose $G=(U \cup V, E)$ is a bipartite graph. For nonempty subsets $U^{\prime} \subseteq U$ and $V^{\prime} \subseteq V$, let $G\left[U^{\prime}, V^{\prime}\right]=\left\{\{u, v\} \in E: u \in U^{\prime}, v \in V^{\prime}\right\}$ be the subgraph of $G$ induced on $U^{\prime}$ and $V^{\prime}$. Set $d\left(U^{\prime}, V^{\prime}\right)=\left|G\left[U^{\prime}, V^{\prime}\right]\right|\left|U^{\prime}\right|^{-1}\left|V^{\prime}\right|^{-1}$ to be the density of $U^{\prime}$ and $V^{\prime}$. For $\varepsilon>0$, we say $G=(U \cup V, E)$ is $\varepsilon$-regular if for all $U^{\prime} \subseteq U,\left|U^{\prime}\right|>\varepsilon|U|$, and $V^{\prime} \subseteq V,\left|V^{\prime}\right|>\varepsilon|V|$, we have ${ }^{1} d\left(U^{\prime}, V^{\prime}\right)=d(U, V) \pm \varepsilon$.

\footnotetext{
${ }^{1}$ For simplicity of calculations in this paper, $s=(a \pm b) t$ is short for $(a-b) t \leq s \leq(a+b) t$.
} 


\subsection{Equivalent conditions for $\varepsilon$-regularity}

We consider the following two conditions for a bipartite graph $G=(U \cup V, E)$ with fixed density $d$ (where, whenever needed, we assume $|U|$ and $|V|$ are sufficiently large). For $0<\varepsilon, \delta \leq 1$, consider

$$
\begin{array}{ll}
\mathbf{G}_{\mathbf{1}}=\mathbf{G}_{\mathbf{1}}(\varepsilon) & G \text { is } \varepsilon \text {-regular. } \\
\mathbf{G}_{\mathbf{2}}=\mathbf{G}_{\mathbf{2}}(\delta) & (i) \operatorname{deg}_{G}(u)=(d \pm \delta)|V| \text { for all but } \delta|U| \text { vertices } u \in U, \\
& (\text { ii }) \operatorname{deg}_{G}\left(u, u^{\prime}\right)=(d \pm \delta)^{2}|V| \text { for all but } \delta|U|^{2} \text { distinct pairs } u, u^{\prime} \in U .
\end{array}
$$

\subsection{1 $\mathrm{G}_{1} \Longleftrightarrow \mathrm{G}_{2}$}

The following fact, called the intersection property, is part of the folklore and is easily proved from the definition of $\varepsilon$-regularity (cf. [5]).

Fact 1.1 (Intersection Property, $\mathbf{G}_{\mathbf{1}} \Longrightarrow \mathbf{G}_{\mathbf{2}}$ ) For all $0<\varepsilon<d / 2, \mathbf{G}_{\mathbf{1}}(\varepsilon) \Longrightarrow \mathbf{G}_{\mathbf{2}}(4 \varepsilon)$. In this sense, $\mathbf{G}_{\mathbf{1}} \Longrightarrow \mathbf{G}_{\mathbf{2}}$.

The following non-trivial theorem was proved by Alon, Duke, Lefmann, Rödl and Yuster in [1] and by Duke, Lefmann and Rödl in [2].

Theorem $1.2\left(\mathbf{G}_{\mathbf{2}} \Longrightarrow \mathbf{G}_{\mathbf{1}}\right)$ For all $\delta>0, \mathbf{G}_{\mathbf{2}}(\delta) \Longrightarrow \mathbf{G}_{\mathbf{1}}\left(16 \delta^{1 / 5}\right)$. In this sense, $\mathbf{G}_{\mathbf{2}} \Longrightarrow$ $\mathrm{G}_{1}$.

We mention that the proof of Theorem 1.2 in [1] (cf. [2]) is elegant and far from obvious. We return to this point momentarily.

Fact 1.1 and Theorem 1.2 give an equivalence between the conditions $\mathbf{G}_{\mathbf{1}}$ and $\mathbf{G}_{\mathbf{2}}$.

Corollary $\mathbf{1 . 3}\left(\mathbf{G}_{\mathbf{1}} \Longleftrightarrow \mathbf{G}_{\mathbf{2}}\right)$ For every $\delta>0$ there exists $\varepsilon>0$ (viz. $\varepsilon=\delta / 4$ ) so that $\mathbf{G}_{\mathbf{1}}(\varepsilon) \Longrightarrow \mathbf{G}_{\mathbf{2}}(\delta)$ and for every $\varepsilon>0$ there exists $\delta>0$ (viz. $\delta=\varepsilon^{5} / 16$ ) so that $\mathbf{G}_{\mathbf{2}}(\delta) \Longrightarrow \mathbf{G}_{\mathbf{1}}(\varepsilon)$. In this sense, $\mathbf{G}_{\mathbf{1}} \Longleftrightarrow \mathbf{G}_{\mathbf{2}}$.

We make the following remark.

Remark 1.4 (Corollary $1.3 \Longrightarrow$ Algorithmic SRL) The original proof of Szemerédi's Regularity Lemma was non-constructive. Alon, Duke, Lefmann, Rödl and Yuster [1] (cf. [2]) subsequently established an algorithmic version of the regularity lemma which efficiently constructs the "regular environment" Szemerédi's lemma provides. The central tool in the proof of the algorithmic version of Szemerédi's lemma is Corollary 1.3. 


\subsubsection{The matrix proof of Theorem 1.2}

We briefly describe the matrix construction which verifies Theorem 1.2. Let $G=$ $(U \cup V, E)$ satisfy $\mathbf{G}_{\mathbf{2}}=\mathbf{G}_{\mathbf{2}}(\delta)$ where we set $\varepsilon=16 \delta^{1 / 5}$. To show $G$ is $\varepsilon$-regular, set $\rho=d^{-1}(1-d)$ and construct $\{-1, \rho\}$-matrix $\mathbf{M}=\left(m_{u v}\right)_{u \in U, v \in V}$ by setting $m_{u v}=\rho \Longleftrightarrow$ $\{u, v\} \in G$. Let $\mathbf{r}_{u}$ denote the row vector associated with $u \in U$. $[2])$

Now, let $U^{\prime} \subseteq U,\left|U^{\prime}\right|>\varepsilon|U|, V^{\prime} \subseteq V,\left|V^{\prime}\right|>\varepsilon|V|$, be given. One may establish (cf.

$$
\left(\frac{d\left(U^{\prime}, V^{\prime}\right)}{d}-1\right)^{2} \leq\left|U^{\prime}\right|^{-2}\left|V^{\prime}\right|^{-1}\left(\sum_{u \in U^{\prime}} \mathbf{r}_{u} \cdot \mathbf{r}_{u}+2 \sum_{\left\{u, u^{\prime}\right\} \in\left[U^{\prime}\right]^{2}} \mathbf{r}_{u} \cdot \mathbf{r}_{u^{\prime}}\right)
$$

where "." denotes scalar product for vectors. The inequality $\left|d\left(U^{\prime}, V^{\prime}\right)-d\right|<\varepsilon$ then follows from manipulating the expression above using the hypothesis $\mathbf{G}_{\mathbf{2}}(\delta)$.

\subsection{Content of this Note}

We work with the following simplified condition ${ }^{2}$

$$
\mathbf{G}_{\mathbf{2}}^{\prime}=\mathbf{G}_{\mathbf{2}}^{\prime}(\delta) \quad \operatorname{deg}\left(u, u^{\prime}\right)=(d \pm \delta)^{2}|V| \text { for all but } \delta|U|^{2} \text { pairs } u, u^{\prime} \in U
$$

Our goal is to prove the following theorem.

Theorem $1.5\left(\mathbf{G}_{\mathbf{2}}^{\prime} \Longrightarrow \mathbf{G}_{\mathbf{1}}\right)$ For all $\varepsilon>0$, there exists $\delta$ so that $\mathbf{G}_{\mathbf{2}}^{\prime}(\delta) \Longrightarrow \mathbf{G}_{\mathbf{1}}(\varepsilon)$.

We note that our result, Theorem 1.5, is a bit weaker than Theorem 1.2 in the sense that our constant $\delta=\delta(\varepsilon)$ is considerably smaller than $\varepsilon^{5} / 16$.

In our proof of Theorem 1.5, we do not appeal to the matrix argument of Section 1.1.2. We show $\mathbf{G}_{\mathbf{2}}^{\prime} \Longrightarrow \mathbf{G}_{\mathbf{1}}$ follows directly from an application of the Szemerédi Regularity Lemma itself.

\section{Proof of Theorem 1.5}

In this section, we prove Theorem 1.5. In our proof, $G=(U \cup V, E)$ always represents a bipartite graph of density $d$ with $m=|U| \leq|V|=n$. We state, up front, that we always assume $m$ is a sufficiently large integer.

Our proof of Theorem 1.5 uses a well-known invariant formulation of Szemerédi's Regularity Lemma. We now present that formulation.

\footnotetext{
${ }^{2}$ As noted by Kohayakawa, Rödl and Skokan [3], statement $(i)$ of condition $\mathbf{G}_{\mathbf{2}}$ is not actually needed. Indeed, as shown in Claim 5.3 of [3], statement $(i)$ of condition $\mathbf{G}_{\mathbf{2}}\left(\delta^{\prime}\right)$ follows from statement (ii) of condition $\mathbf{G}_{\mathbf{2}}(\delta)$, for a suitable $\delta$, using a Cauchy-Schwarz argument.
} 


\subsection{An Invariant of Szemerédi's Regularity Lemma}

Let $G=(U \cup V, E)$ be a bipartite graph. For an integer $t$, we define a $t$-equitable partition $V(G)$ as a pair of partitions $U=U_{1} \cup \ldots \cup U_{t}, V=V_{1} \cup \ldots \cup V_{t}$, where

$$
\left\lfloor\frac{m}{t}\right\rfloor=\left\lfloor\frac{|U|}{t}\right\rfloor \leq\left|U_{1}\right| \leq \ldots \leq\left|U_{t}\right| \leq\left\lceil\frac{|U|}{t}\right\rceil=\left\lceil\frac{m}{t}\right\rceil
$$

and

$$
\left\lfloor\frac{n}{t}\right\rfloor=\left\lfloor\frac{|V|}{t}\right\rfloor \leq\left|V_{1}\right| \leq \ldots \leq\left|V_{t}\right| \leq\left\lceil\frac{|V|}{t}\right\rceil=\left\lceil\frac{n}{t}\right\rceil .
$$

In all that follows, $o(1) \rightarrow 0$ as $m \rightarrow \infty$. Thus, in the remainder of this paper, we may say that for each $1 \leq i \leq t$,

$$
\left|U_{i}\right|=\frac{m}{t}(1 \pm o(1)), \quad\left|V_{i}\right|=\frac{n}{t}(1 \pm o(1)) .
$$

For convience of notation, we write $G^{i j}=G\left[U_{i}, V_{j}\right]$ and $d_{i j}=d_{G}\left(U_{i}, V_{j}\right), 1 \leq i, j \leq t$.

For $\varepsilon_{0}>0$, we say a $t$-equitable partition $U=U_{1} \cup \ldots \cup U_{t}, V=V_{1} \cup \ldots \cup V_{t}$, is $\varepsilon_{0}$-regular if all but $\varepsilon_{0} t^{2}$ biparite graphs $G^{i j}, 1 \leq i, j \leq t$, are $\varepsilon_{0}$-regular.

Theorem 2.1 (Regularity Lemma) For every $\varepsilon_{0}>0$ and positive integer $t_{0}$, there exists $N_{0}$ and $T_{0}$ so that every bipartite graph $G=(U \cup V, E)$ with $n=|V| \geq m=|U| \geq N_{0}$ admits a t-equitable, $\varepsilon_{0}$-regular partition $U=U_{1} \cup \ldots \cup U_{t}, V=V_{1} \cup \ldots \cup V_{t}$, for $t_{0} \leq t \leq T_{0}$.

Note that the proof of Theorem 2.1 takes an existing parition and refines it. As a result, clusters $U_{i}$ are subsets of $U$ and clusters $V_{j}$ are subsets of $V$.

\section{$2.2 \varepsilon_{0}$-regular partitions and $\mathbf{G}_{2}^{\prime}(\delta)$}

The following statement, expressed in Proposition 2.2, will imply Theorem 1.5 almost immediately.

Proposition 2.2 Let $d, \varepsilon_{0}>0$ be given along with an integer $t$. Let $0<\delta<\varepsilon_{0} / t^{2}$ be given. Let $G=(U \cup V, E)$ be a bipartite graph of density d satisfying $\mathbf{G}_{\mathbf{2}}^{\prime}(\delta)$ and let $U=U_{1} \cup \ldots \cup U_{t}, V=V_{1} \cup \ldots \cup V_{t}$, be an $\varepsilon_{0}$-regular, $t$-equitable partition of $V(G)$. Then, at most $5 \varepsilon_{0}^{1 / 3} t^{2}$ pairs $U_{i}, V_{j}, 1 \leq i, j \leq t$, fail to both be $\varepsilon_{0}$-regular and satisfy $d_{i j}=d \pm 5 \varepsilon_{0}^{1 / 3}$.

Note that Proposition 2.2 essentially says that with appropriate constants ${ }^{3}$, property $\mathbf{G}_{\mathbf{2}}^{\prime}(\delta)$ forces the density $d$ to be preserved throughout almost all bipartite graphs $G^{i j}$, $1 \leq i, j \leq t$, of the partition. As almost all bipartite graphs $G^{i j}, 1 \leq i, j \leq t$, are also $\varepsilon_{0}$-regular, $\varepsilon_{0} \ll \varepsilon$, the preserved densities quickly imply the $\varepsilon$-regularity of $G$.

\footnotetext{
${ }^{3}$ Here, one may think of the hierarchy " $d \gg \varepsilon_{0} \gg 1 / t \gg \delta$ ".
} 


\subsection{Proof of Theorem 1.5}

Before proceeding to the proof of Theorem 1.5, we begin by describing the constants involved, the setup we use and a few preparations we make. We begin with the constants.

\subsubsection{The Constants}

Let $d, \varepsilon>0$ be given. To define the promised constant $\delta>0$, set auxiliary constants

$$
\varepsilon_{0}=\left(d^{3} \varepsilon^{15}\right) / 20^{3}
$$

and $t_{0}=1$. Let $T_{0}=T_{0}\left(\varepsilon_{0}, 1\right)$ be the constant guaranteed by Theorem 2.1. Define $\delta=\varepsilon_{0} / 2 T_{0}^{2}$.

\subsubsection{The Setup}

Let $G=(U \cup V, E)$ be a bipartite graph of density $d$ satisfying $\mathbf{G}_{\mathbf{2}}^{\prime}(\delta)$ where the integers $|V|=n \geq m=|U|$ are sufficiently large.

We show $G$ is $\varepsilon$-regular. To that end, let $U^{\prime} \subseteq U, V^{\prime} \subseteq V,\left|U^{\prime}\right|>\varepsilon m,\left|V^{\prime}\right|>\varepsilon n$, be given. We show $d_{G}\left(U^{\prime}, V^{\prime}\right)=d \pm \varepsilon$.

\subsubsection{Preparations}

We begin by applying Theorem 2.1 to $G$. With auxiliary constants $\varepsilon_{0}=\left(d^{3} \varepsilon^{15} / 20^{3}\right)$ and $t_{0}=1$, Theorem 2.1 guarantees constants $T_{0}=T_{0}\left(\varepsilon_{0}, 1\right)$ and $N_{0}=N_{0}\left(\varepsilon_{0}, 1\right)$. With $n=|V| \geq|U|=m \geq N_{0}$, we may apply Theorem 2.1 to $G$ to obtain an $\varepsilon_{0}$-regular, $t$-equitable partition $U=U_{1} \cup \ldots \cup U_{t}, V=V_{1} \cup \ldots \cup V_{t}$, where $1=t_{0} \leq t \leq T_{0}$. Note, importantly, that $T_{0}=T_{0}\left(\varepsilon_{0}, 1\right)$ is precisely the same constant we saw above when we set $\delta=\varepsilon_{0} /\left(2 T_{0}^{2}\right)$. In this way, we are ensured $\delta<\varepsilon_{0} / t^{2}$.

We now wish to apply Proposition 2.2 to $G$ and its $\varepsilon_{0}$-regular, $t$-equitable partition $U=U_{1} \cup \ldots \cup U_{t}, V=V_{1} \cup \ldots \cup V_{t}$, obtained above. Note that we may apply Proposition 2.2 (since $\delta<\varepsilon_{0} / t^{2}$ ). Applying Proposition 2.2, we are guaranteed that all but $5 \varepsilon_{0}^{1 / 3} t^{2}$ pairs $U_{i}, V_{j}, 1 \leq i, j \leq t$, are $\varepsilon_{0}$-regular and satisfy $d_{i j}=d \pm 5 \varepsilon_{0}^{1 / 3}$.

Now, define graph $G_{0}$ to have vertex set $[t] \times[t]$ where

$$
G_{0}=\left\{(i, j) \in[t] \times[t]: G^{i j} \text { is } \varepsilon_{0} \text {-regular with density } d_{i j}=d \pm 5 \varepsilon_{0}^{1 / 3}\right\} .
$$

Set $G_{0}^{C}=([t] \times[t]) \backslash G_{0}$.

In the notation $G_{0}^{C}$ above, Proposition 2.2 precisely says

$$
\left|G_{0}^{C}\right| \leq 5 \varepsilon_{0}^{1 / 3} t^{2} .
$$


For $1 \leq i \leq t$, set $U_{i}^{\prime}=U^{\prime} \cap U_{i}$ and $V_{i}^{\prime}=V^{\prime} \cap V_{i}$. For $1 \leq i, j \leq t$, define the graph $B$ to have vertex set $[t] \times[t]$ where

$$
B=\left\{(i, j) \in[t] \times[t]:\left|U_{i}^{\prime}\right|>\varepsilon_{0}\left|U_{i}\right| \text { and }\left|V_{i}^{\prime}\right|>\varepsilon_{0}\left|V_{i}\right|\right\} .
$$

Set $B^{C}=[t] \times[t] \backslash B$.

\subsubsection{Proof of Theorem 1.5}

Recall we are given $U^{\prime} \subseteq U, V^{\prime} \subseteq V,\left|U^{\prime}\right|>\varepsilon m,\left|V^{\prime}\right|>\varepsilon n$, and we want to show $d_{G}\left(U^{\prime}, V^{\prime}\right)=d \pm \varepsilon$, or equivalently,

$$
\left|G\left[U^{\prime}, V^{\prime}\right]\right| \geq(d-\varepsilon)\left|U^{\prime}\right|\left|V^{\prime}\right|, \text { and }
$$

$\left|G\left[U^{\prime}, V^{\prime}\right]\right| \leq(d+\varepsilon)\left|U^{\prime}\right|\left|V^{\prime}\right|$. As both statements have virtually the same proof with identical calculations, we only show (5).

Observe

$$
\begin{aligned}
\left|G\left[U^{\prime}, V^{\prime}\right]\right| & =\sum_{1 \leq i, j \leq t}\left|G\left[U_{i}^{\prime}, V_{j}^{\prime}\right]\right|=\sum_{(i, j) \in G_{0} \cap B}\left|G\left[U_{i}^{\prime}, V_{j}^{\prime}\right]\right|+\sum_{(i, j) \notin G_{0} \cap B}\left|G\left[U_{i}^{\prime}, V_{j}^{\prime}\right]\right| \\
& \geq \sum_{(i, j) \in G_{0} \cap B}\left|G\left[U_{i}^{\prime}, V_{j}^{\prime}\right]\right| \geq \sum_{(i, j) \in G_{0} \cap B}\left(d-5 \varepsilon_{0}^{1 / 3}\right)\left|U_{i}^{\prime}\right|\left|V_{j}^{\prime}\right| .
\end{aligned}
$$

On account of $\varepsilon_{0}=\left(d^{3} \varepsilon^{15} / 20^{3}\right)$ (cf. (2)), we see

$$
\left(d-5 \varepsilon_{0}^{1 / 3}\right)=d\left(1-\frac{5 \varepsilon_{0}^{1 / 3}}{d}\right) \geq d\left(1-\varepsilon^{2}\right) .
$$

Thus, we conclude

$$
\left|G\left[U^{\prime}, V^{\prime}\right]\right| \geq d\left(1-\varepsilon^{2}\right) \sum_{(i, j) \in G_{0} \cap B}\left|U_{i}^{\prime}\right|\left|V_{j}^{\prime}\right|
$$

Observe

$$
\begin{gathered}
\sum_{(i, j) \in G_{0} \cap B}\left|U_{i}^{\prime}\right|\left|V_{j}^{\prime}\right| \geq \sum_{1 \leq i, j \leq t}\left|U_{i}^{\prime}\right|\left|V_{j}^{\prime}\right|-\sum_{(i, j) \in G_{0}^{C}}\left|U_{i}^{\prime}\right|\left|V_{j}^{\prime}\right|-\sum_{(i, j) \in B^{C}}\left|U_{i}^{\prime}\right|\left|V_{j}^{\prime}\right| \\
=\left|U^{\prime}\right|\left|V^{\prime}\right|-\sum_{(i, j) \in G_{0}^{C}}\left|U_{i}^{\prime}\right|\left|V_{j}^{\prime}\right|-\sum_{(i, j) \in B^{C}}\left|U_{i}^{\prime}\right|\left|V_{j}^{\prime}\right| .
\end{gathered}
$$

Now, $\left|G_{0}^{C}\right|<5 \varepsilon_{0}^{1 / 3} t^{2}$ (cf. (3)). By (4), each term in the last sum above is at most $\varepsilon_{0}\left|U_{i}\right|\left|V_{i}\right|=\varepsilon_{0}(1+o(1)) \frac{m n}{t^{2}} \leq 2 \varepsilon_{0} \frac{m n}{t^{2}}$ (cf. (1)). We therefore see

$$
\sum_{(i, j) \in G_{0} \cap B}\left|U_{i}^{\prime}\right|\left|V_{j}^{\prime}\right| \geq\left|U^{\prime}\right|\left|V^{\prime}\right|-10 \varepsilon_{0}^{1 / 3} m n-2 \varepsilon_{0} m n=\left|U^{\prime}\right|\left|V^{\prime}\right|\left[1-\frac{10 \varepsilon_{0}^{1 / 3} m n+2 \varepsilon_{0} m n}{\left|U^{\prime}\right|\left|V^{\prime}\right|}\right] .
$$


As $\left|U^{\prime}\right|>\varepsilon m$ and $\left|V^{\prime}\right|>\varepsilon n$ and $\varepsilon_{0}=\left(d^{3} \varepsilon^{15} / 20^{3}\right)$ from (2), we conclude

$$
\sum_{(i, j) \in G_{0} \cap B}\left|U_{i}^{\prime}\right|\left|V_{j}^{\prime}\right| \geq\left|U^{\prime}\right|\left|V^{\prime}\right|\left(1-\varepsilon^{3}-\varepsilon^{13}\right) \geq\left|U^{\prime}\right|\left|V^{\prime}\right|\left(1-\varepsilon^{2}\right) .
$$

Combining (6) and (7), we see

$$
\left|G\left[U^{\prime}, V^{\prime}\right]\right| \geq d\left(1-\varepsilon^{2}\right)^{2}\left|U^{\prime}\right|\left|V^{\prime}\right| \geq d\left(1-2 \varepsilon^{2}\right)\left|U^{\prime}\right|\left|V^{\prime}\right| \geq(d-\varepsilon)\left|U^{\prime}\right|\left|V^{\prime}\right| .
$$

This proves (5) and hence Theorem 1.5.

\subsection{Proof of Proposition 2.2}

Let $0<d \leq 1, \varepsilon_{0}>0$ and integer $t$ be given. Let $0<\delta<\varepsilon_{0} / t^{2}$ be given. Let $G=(U \cup V, E)$ be a bipartite graph of density $d$ satisfying $\mathbf{G}_{\mathbf{2}}^{\prime}(\delta)$ and let $U=U_{1} \cup \ldots \cup U_{t}$, $V=V_{1} \cup \ldots \cup V_{t}$, be an $\varepsilon_{0}$-regular, $t$-equitable partition of $V(G)$. We show all but $5 \varepsilon_{0}^{1 / 3} t^{2}$ pairs $U_{i}, V_{j}, 1 \leq i, j \leq t$, span $\varepsilon_{0}$-regular bipartite graphs $G^{i j}$ of density $d_{i j}=d \pm 5 \varepsilon_{0}^{1 / 3}$.

By definition of $\varepsilon_{0}$-regular, $t$-equitable partition, we have all but $\varepsilon_{0} t^{2}$ pairs $U_{i}, V_{j}$, $1 \leq i, j \leq t$, spanning $\varepsilon_{0}$-regular bipartite graphs $G^{i j}$. Thus, it suffices to show all but $4 \varepsilon_{0}^{1 / 3} t^{2}$ pairs $U_{i}, V_{j}, 1 \leq i, j \leq t$, span bipartite graphs $G^{i j}$ of density $d_{i j}=d \pm 5 \varepsilon_{0}^{1 / 3}$.

The following two claims prove Proposition 2.2 almost immediately.

\section{Claim 2.3}

$$
\sum_{1 \leq i, j \leq t} d_{i j} \geq d t^{2}(1-o(1))
$$

\section{Claim 2.4}

$$
\sum_{1 \leq i, j \leq t} d_{i j}^{2}<d^{2} t^{2}\left(1+18 \varepsilon_{0}\right) .
$$

Indeed, we now prove Proposition 2.2 from Claims 2.3 and 2.4 using the following well-known fact (cf. [3]).

Fact 2.5 (Approximate Cauchy-Schwarz) For every $\zeta>0,0<\gamma \leq \zeta^{3} / 3$ and nonnegative reals $a_{1}, \ldots, a_{r}$ satisfying

1. $\sum_{j=1}^{r} a_{j} \geq(1-\gamma) r a$, and

2. $\sum_{j=1}^{r} a_{j}^{2}<(1+\gamma) r a^{2}$,

we have

$$
\left|\left\{j:\left|a-a_{j}\right|<\zeta a\right\}\right|>(1-\zeta) r .
$$

With $\gamma=18 \varepsilon_{0}, \zeta=\left(54 \varepsilon_{0}\right)^{1 / 3}, r=t^{2}$ and $\left\{a_{1}, \ldots, a_{r}\right\}=\left\{d_{i j}: 1 \leq i, j \leq t\right\}$ we see Claim 2.3 satisfies (1) of Fact 2.5 and Claim 2.4 satisfies (2) of Fact 2.5. By Fact 2.5, we see at most $\zeta t^{2}=\left(54 \varepsilon_{0}\right)^{1 / 3} t^{2} \leq 4 \varepsilon_{0}^{1 / 3} t^{2}$ pairs $1 \leq i, j \leq t$, satisfy $d_{i j}=d(1 \pm \zeta)$ and so $d_{i j}=d \pm \zeta$ and finally $d_{i j}=d \pm 4 \varepsilon_{0}^{1 / 3}$. The proof of Proposition 2.2 will then be complete upon the proofs of Claims 2.3 and 2.4. 


\subsubsection{Proof of Claim 2.3}

Recall $G$ has density $d$. Consequently,

$$
d m n=|G|=\sum_{1 \leq i, j \leq t}\left|G^{i j}\right|=\sum_{1 \leq i, j \leq t} d_{i j}\left|U_{i}\right|\left|V_{i}\right|=\frac{m n}{t^{2}}(1+o(1)) \sum_{1 \leq i, j \leq t} d_{i j} .
$$

Claim 2.3 now follows.

\subsubsection{Proof of Claim 2.4}

We begin by giving some notation.

Notation and Preparation.

Set

$$
\Gamma=\left\{\left\{u, u^{\prime}\right\} \in[U]^{2}: \operatorname{deg}_{G}\left(u, u^{\prime}\right)=(d \pm \delta)^{2} n\right\}, \quad \Gamma^{C}=[U]^{2} \backslash \Gamma .
$$

For $1 \leq i \leq t$, set

$$
\Gamma_{i}=\Gamma \cap\left[U_{i}\right]^{2}, \quad \Gamma_{i}^{C}=\left[U_{i}\right]^{2} \backslash \Gamma=\Gamma^{C} \cap\left[U_{i}\right]^{2} .
$$

Note that since $G$ satisfies $\mathbf{G}_{\mathbf{2}}^{\prime}(\delta)$, we may conclude

$$
\left|\Gamma^{C}\right|<\delta m^{2}, \quad\left|\Gamma_{i}^{C}\right| \leq\left|\Gamma^{C}\right|<\delta m^{2}
$$

where the last inequality is purely greedy.

Set $I_{\varepsilon_{0}}$ to be the bipartite graph with bipartition $[t] \times[t]$ where

$$
(i, j) \in I_{\varepsilon_{0}} \Longleftrightarrow G^{i j} \text { is } \varepsilon_{0} \text {-irregular. }
$$

Set $S$ to be the bipartite graph with bipartition $[t] \times[t]$ where

$$
(i, j) \in S \Longleftrightarrow(i, j) \notin I_{\varepsilon_{0}} \text { and } d_{i j}<\sqrt{\varepsilon_{0}} \text {. }
$$

Let

$$
D=[t] \times[t] \backslash\left(I_{\varepsilon_{0}} \cup S\right) .
$$

As $\left|I_{\varepsilon_{0}}\right|<\varepsilon_{0} t^{2}$ and since $U_{i}$ and $V_{j},(i, j) \in S$, span few edges, we have the following fact.

\section{Fact 2.6}

$$
\sum_{(i, j) \in D} d_{i j}^{2} \geq \sum_{1 \leq i, j \leq t} d_{i j}^{2}-2 \varepsilon_{0} t^{2}
$$

For $(i, j) \in D$, set

$$
\Gamma_{i j}=\left\{\left\{u, u^{\prime}\right\} \in\left[U_{i}\right]^{2}: \operatorname{deg}_{G^{i j}}\left(u, u^{\prime}\right)=\left(d_{i j} \pm \varepsilon_{0}\right)^{2}\left|V_{i}\right|\right\}
$$


For $(i, j) \in D, G^{i j}$ is $\varepsilon_{0}$-regular with density $d_{i j}>\sqrt{\varepsilon_{0}}>2 \varepsilon_{0}$. Thus, from Fact 1.1 , we see

$$
\left|\left[U_{i}\right]^{2} \backslash \Gamma_{i j}\right|<4 \varepsilon_{0}\left|U_{i}\right|^{2}
$$

This concludes our notation and preparations. We now proceed to the proof of Claim 2.4.

Proof of Claim 2.4.

We double-count the quantity $\sum_{1 \leq i, j \leq t} \sum_{\left\{u, u^{\prime}\right\} \in\left[U_{i}\right]^{2}} \operatorname{deg}_{G^{i j}}\left(u, u^{\prime}\right)$. In particular, we show the following two facts.

Fact 2.7

$$
\sum_{1 \leq i, j \leq t} \sum_{\left\{u, u^{\prime}\right\} \in\left[U_{i}\right]^{2}} \operatorname{deg}_{G^{i j}}\left(u, u^{\prime}\right) \leq \frac{n m^{2}}{2 t}\left(d^{2}+5 \delta t^{2}\right)
$$

Fact 2.8

$$
\sum_{1 \leq i, j \leq t} \sum_{\left\{u, u^{\prime}\right\} \in\left[U_{i}\right]^{2}} \operatorname{deg}_{G^{i j}}\left(u, u^{\prime}\right) \geq\left(1-9 \varepsilon_{0}\right) \frac{n m^{2}}{2 t^{3}}\left[\left(\sum_{1 \leq i, j \leq t} d_{i j}^{2}\right)-4 \varepsilon_{0} t^{2}\right] .
$$

We see Claim 2.4 follows quickly from Facts 2.7 and 2.8. Indeed, comparing the two facts, we get

$$
\frac{n m^{2}}{2 t}\left(d^{2}+5 \delta t^{2}\right) \geq\left(1-9 \varepsilon_{0}\right) \frac{n m^{2}}{2 t^{3}}\left[\left(\sum_{1 \leq i, j \leq t} d_{i j}^{2}\right)-4 \varepsilon_{0} t^{2}\right]
$$

which implies $\left(\sum_{1 \leq i, j \leq t} d_{i j}^{2}\right) \leq d^{2} t^{2}+5 \delta t^{4}+13 \varepsilon_{0} t^{2}$. On account of $\delta \leq \varepsilon_{0} / t^{2}$, we further conclude $\left(\sum_{1 \leq i, j \leq t} d_{i j}^{2}\right) \leq d^{2} t^{2}+18 \varepsilon_{0} t^{2}$ which proves Claim 2.4. It therefore suffices to prove the two facts above.

\section{Proof of Fact 2.7.}

Observe

$\sum_{1 \leq i, j \leq t} \sum_{\left\{u, u^{\prime}\right\} \in\left[U_{i}\right]^{2}} \operatorname{deg}_{G^{i j}}\left(u, u^{\prime}\right)=\sum_{1 \leq i \leq t} \sum_{\left\{u, u^{\prime}\right\} \in\left[U_{i}\right]^{2}} \sum_{1 \leq j \leq t} \operatorname{deg}_{G^{i j}}\left(u, u^{\prime}\right)=\sum_{1 \leq i \leq t} \sum_{\left\{u, u^{\prime}\right\} \in\left[U_{i}\right]^{2}} \operatorname{deg}_{G}\left(u, u^{\prime}\right)$.

Recalling $\left[U_{i}\right]^{2}=\Gamma_{i} \cup \Gamma_{i}^{C}$ is a partition (cf. (9)), $1 \leq i \leq t$, we see

$$
\sum_{1 \leq i, j \leq t} \sum_{\left\{u, u^{\prime}\right\} \in\left[U_{i}\right]^{2}} \operatorname{deg}_{G^{i j}}\left(u, u^{\prime}\right)=\sum_{1 \leq i \leq t} \sum_{\left\{u, u^{\prime}\right\} \in \Gamma_{i}} \operatorname{deg}_{G}\left(u, u^{\prime}\right)+\sum_{1 \leq i \leq t} \sum_{\left\{u, u^{\prime}\right\} \in \Gamma_{i}^{C}} \operatorname{deg}_{G}\left(u, u^{\prime}\right) .
$$

Then, according to (8) and (9)

$$
\begin{gathered}
\sum_{1 \leq i, j \leq t} \sum_{\left\{u, u^{\prime}\right\} \in\left[U_{i}\right]^{2}} \operatorname{deg}_{G^{i j}}\left(u, u^{\prime}\right) \leq \sum_{1 \leq i \leq t} \sum_{\left\{u, u^{\prime}\right\} \in \Gamma_{i}}(d+\delta)^{2}|V|+\sum_{1 \leq i \leq t} \sum_{\left\{u, u^{\prime}\right\} \in \Gamma_{i}^{C}}|V| \\
\leq n\left((d+\delta)^{2} \sum_{1 \leq i \leq t}\left|\Gamma_{i}\right|+\sum_{1 \leq i \leq t}\left|\Gamma_{i}^{C}\right|\right) \leq n\left((d+\delta)^{2} \sum_{1 \leq i \leq t}\left(\begin{array}{c}
\left|U_{i}\right| \\
2
\end{array}\right)+\sum_{1 \leq i \leq t}\left|\Gamma_{i}^{C}\right|\right) .
\end{gathered}
$$


From (10), we conclude

$$
\sum_{1 \leq i, j \leq t} \sum_{\left\{u, u^{\prime}\right\} \in\left[U_{i}\right]^{2}} \operatorname{deg}_{G^{i j}}\left(u, u^{\prime}\right) \leq n\left[(d+\delta)^{2} t\left(\frac{1}{2}+o(1)\right)\left(\frac{m}{t}\right)^{2}+\delta t m^{2}\right] .
$$

Fact 2.7 now follows.

Proof of Fact 2.8.

Since $D \subseteq[t] \times[t]($ cf. $(11))$ and $\Gamma_{i j} \subseteq\left[U_{i}\right]^{2}$ (cf. (12)), we see

$$
\begin{gathered}
\sum_{1 \leq i, j \leq t} \sum_{\left\{u, u^{\prime}\right\} \in\left[U_{i}\right]^{2}} \operatorname{deg}_{G^{i j}}\left(u, u^{\prime}\right) \geq \sum_{(i, j) \in D} \sum_{\left\{u, u^{\prime}\right\} \in \Gamma_{i j}} \operatorname{deg}_{G^{i j}}\left(u, u^{\prime}\right) \\
\sum_{(i, j) \in D} \sum_{\left\{u, u^{\prime}\right\} \in \Gamma_{i j}}\left(d_{i j}-\varepsilon_{0}\right)^{2}\left|V_{j}\right|=(1-o(1)) \frac{n}{t} \sum_{(i, j) \in D} \sum_{\left\{u, u^{\prime}\right\} \in \Gamma_{i j}}\left(d_{i j}-\varepsilon_{0}\right)^{2} \\
\geq \frac{n}{t} \sum_{(i, j) \in D}\left(d_{i j}^{2}-2 \varepsilon_{0}\right)\left|\Gamma_{i j}\right| .
\end{gathered}
$$

From (13), we thus see

$$
\begin{aligned}
& \sum_{1 \leq i, j \leq t} \sum_{\left\{u, u^{\prime}\right\} \in\left[U_{i}\right]^{2}} \operatorname{deg}_{G^{i j}}\left(u, u^{\prime}\right) \geq \frac{n}{t} \sum_{(i, j) \in D}\left(d_{i j}^{2}-2 \varepsilon_{0}\right)\left[\left(\begin{array}{c}
\left|U_{i}\right| \\
2
\end{array}\right)-4 \varepsilon_{0}\left|U_{i}\right|^{2}\right] \\
= & \left(1-9 \varepsilon_{0}\right) \frac{n m^{2}}{2 t^{3}} \sum_{(i, j) \in D}\left(d_{i j}^{2}-2 \varepsilon_{0}\right)=\left(1-9 \varepsilon_{0}\right) \frac{n m^{2}}{2 t^{3}}\left[\sum_{(i, j) \in D} d_{i j}^{2}-\sum_{(i, j) \in D} 2 \varepsilon_{0}\right] .
\end{aligned}
$$

However, from Fact 2.6 and the fact that $|D| \leq t^{2}$, we see Fact 2.7 follows.

\section{References}

[1] N. Alon, R. Duke, H. Lefmann, V. Rödl and R. Yuster, The algorithmic aspects of the Regularity Lemma (II), J. Algorithms 16 (1994), no. 1, pp 80-109.

[2] R. Duke, H. Lefmann and V. Rödl, A fast algorithm for computing the frequencies of subgraphs in a given graph, SIAM J. Comp. 24 (1995), pp 598-620.

[3] Y. Kohayakawa, V. Rödl and J. Skokan, Quasi-randomness, hypergraphs and conditions for regularity, J. Combin. Theory, Ser. A 97 (2002), no 2, pp 307-352.

[4] J. Komlós, A. Shoukoufandeh, M. Simonovits, E. Szemerédi, The regularity lemma and its applications in graph theory, Theoretical aspects of computer science (Teheran 2000), Lecture Notes in Comput. Sci. 2292, (2002), 84-112.

[5] J. Komlós and M. Simonovits, Szemerédi's Regularity Lemma and its applications in graph theory, in "Combinatorics, Paul Erdős is Eighty" (D. Miklós, V. T. Sós, and T. Szönyi, Eds.), Bolayi Society Mathematical Studies, Vol. 2, Budapest, (1996), 295-352. 
[6] J. Skokan and L. Thoma, Bipartite subgraphs and quasi-randomness, accepted to Graphs and Combinatorics.

[7] E. Szemerédi, Regular partitions of graphs, in "Problemes Combinatoires et Theorie des Graphes, Proc. Colloque Inter. CNRS" (J. C. Bermond, J. C. Fournier, M. Las Vergnas, and D. Sotteau, Eds.), CNRS, Paris, (1978), 399-401. 